\title{
Signal Confidence Limits from a Neural Network Data Analysis ${ }^{1}$
}

\author{
Bernd A. Berg $2,3,4,5$ and Jürgen Riedler ${ }^{2,4,6,7}$
}

\begin{abstract}
This paper deals with a situation of some importance for the analysis of experimental data via Neural Network (NN) or similar devices: Let $N$ data be given, such that $N=N_{s}+N_{b}$, where $N_{s}$ is the number of signals, $N_{b}$ the number of background events, both unknown. Assume that a NN has been trained, such that it will tag signals with efficiency $F_{s},\left(0<F_{s}<1\right)$ and background data with $F_{b},\left(0<F_{b}<1\right)$. Applying the NN yields $N^{Y}$ tagged events. We demonstrate that the knowledge of $N^{Y}$ is sufficient to calculate confidence bounds for the signal likelihood, which have the same statistical interpretation as the Clopper-Pearson bounds for the well-studied case of direct signal observation.

Subsequently, we discuss rigorous bounds for the a-posteriori distribution function of the signal probability, as well as for the (closely related) likelihood that there are $N_{s}$ signals in the data. We compare them with results obtained by starting off with a maximum entropy type assumption for the a-priori likelihood that there are $N_{s}$ signals in the data and applying the Bayesian theorem. Difficulties are encountered with the latter method.
\end{abstract}

\footnotetext{
${ }^{1}$ This research was partially funded by the Department of Energy under contract DE-FG05-87ER40319 and by the Austrian Ministry of Science.

${ }^{2}$ Department of Physics, The Florida State University, Tallahassee, FL 32306, USA.

${ }^{3}$ Supercomputer Computations Research Institute, Tallahassee, FL 32306, USA.

${ }^{4}$ Zentrum für Interdisziplinäre Forschung (ZIF), Wellenberg 1, D-33615 Bielefeld, Germany.

${ }^{5}$ E-mail: berg@hep.fsu.edu

${ }^{6}$ Institut für Kernphysik, Technische Universität Wien, A-1040 Vienna, Austria.

${ }^{7}$ E-mail: juri@kph.tuwien.ac.at
} 


\section{Introduction}

Let us assume that $N_{s}$ signals are observed in $N$ data

$$
N=N_{s}+N_{b}
$$

where $N_{b}$ is the number of background events. We denote the a-priori unknown signal likelihood by $p$. Relying on the binomial distribution, Clopper and Pearson [1] derived a method, which allows to calculate rigorous confidence bounds on $p$, given $N_{s}$ and $N$. Now, in modern physics, in particular high energy physics experiments, it happens quite often that signal and background events belong to overlapping probability densities in a multidimensional parameter space. In such situations signals can only be identified in a statistical sense. Typically, some method may allow to tag signals and background events with different efficiencies: $F_{s}$ for the signals $\left(0<F_{s}<1\right)$ and $F_{b}$ for the background events $\left(0<F_{b}<1\right)$. Instead of observing $N_{s}$ signals we only get

$$
N^{Y} \text { tagged data. }
$$

The question is, what confidence limits on the signal likelihood are then implied? We proof, and illustrate in some detail, that the Clopper-Pearson method can be generalized accordingly.

In particular, we have high energy physics experimental data in mind, where tagging may be provided by traditional cuts or by applying some NN [2, 3, 4, 5, 6] technique. To give an example, figure 5 of Ref.[6] depicts values for neural network efficiencies, $F_{s}(Y)$ and $F_{b}(Y)$, which one may expect to occur for identifying $t \bar{t}$-events in the All-Jets channel [7]. Running the network on all $N$ data assigns to each event a value of the network function $Y_{n}, n=1, \ldots, N$. For a fixed choice of $Y$, the network returns $N^{Y}$ events with $Y_{n} \leq Y$. An additional problem in real applications may be that the efficiencies $F_{s}$ and $F_{b}$ are not exact either. However, as outlined in the conclusions, we think that this difficulty may be overcome by the bootstrap approach [8]. 
In the next section we explain and generalize the Clopper-Pearson approach. A number of illustrations focus on the small number of $N=10$ data, because then the statistical meaning of the confidence bounds becomes most transparent. In section 3 we deal with the limit of a small number of signals hidden in a large data set. Two instructive sets of network efficiencies are chosen, to demonstrate how the general equations are expected to work in practice.

Section 4 considers a-posteriori distribution functions. (i) For the signal probability

$$
F(p)=\int_{0}^{p} \rho\left(p^{\prime}\right) d p^{\prime}
$$

where $\rho(p)$ is the probability density of $p$. (ii) For the likelihood that there are $N_{s}$ signals in the data set

$$
F\left(N_{s}\right)=\sum_{k=0}^{N_{s}} P(k),
$$

where $P\left(N_{s}\right)$ is the probability that there are $N_{s}$ signals in the data data. Rigorous lower and upper bounds are provided. For the examples of section 3 those bounds are close together, such that useful approximations of the true a-posteriori distribution functions result. In section 5 these results are compared with constructing the $F\left(N_{s}\right)$ distribution function and its $P\left(N_{s}\right)$ probability density with the Bayesian method under the maximum entropy assumption that each $N_{s}$ is, a-priori, equally likely. One of the obtained results, and hence its a-priori assumption, is in violation to an exact bound. Conclusions follow in the final section 6 .

\section{From Neural Network Output to Confidence Limits}

Let $p$ be the (unknown) exact likelihood that a data point is a signal. The probability to observe $N_{s}$ signals within $N$ measurements is given by the binomial probability density

$$
b\left(N_{s} \mid N, p\right)=\left(\begin{array}{c}
N \\
N_{s}
\end{array}\right) p^{N_{s}} q^{N-N_{s}}, q=1-p .
$$


We are faced with the inverse problem: if $N_{s}$ signals are observed, what is the confidence to rule out certain $p$ ? Assume that probabilities $p_{-}^{c}$ and $p_{+}^{c}$ are given. Clopper and Pearson [1] define corresponding lower $p_{-}$and upper $p_{+}$bounds as solutions of the equations

$$
p_{-}^{c}=\sum_{k=N_{S}}^{N} b\left(k \mid N, p_{-}\right) \quad \text { and } \quad p_{+}^{c}=\sum_{k=0}^{N_{s}} b\left(k \mid N, p_{+}\right)
$$

with the additional convention $p_{-}=0$ for $N_{s}=0$ and $p_{+}=1$ for $N_{s}=N$. Figure 1 illustrates, how $p_{-}$and $p_{+}$are obtained as parameters of the binomial distributions which yield the areas $p_{-}^{c}$ and $p_{+}^{c}$ as indicated. For this figure we have chosen $N=26 k$ and $N_{s}=130$, in the ballpark of values which will interest us in the next section. Here and in the following binomial coefficients have been calculated relying on Fortran routines of [9].

The precise meaning of the bounds (2) is as follows: $p_{-}$is the largest number such that (for every feasible $p$ ) the probability for $p<p_{-}$is less than $p_{-}^{c}$. Correspondingly, $p_{+}$is the smallest number such that the probability for $p>p_{+}$is less than $p_{+}^{c}$. The other way round,

$$
p \geq p_{-} \text {with likelihood } P_{-}^{c}(p) \geq\left(1-p_{-}^{c}\right)
$$

and

$$
p \leq p_{+} \text {with likelihood } P_{+}^{c}(p) \geq\left(1-p_{+}^{c}\right)
$$

Therefore, for $p_{-}<p_{+}$we find

$$
p \in\left[p_{-}, p_{+}\right] \text {with likelihood } P^{c}(p)=P_{-}^{c}+P_{+}^{c}-1 \geq\left(1-p_{-}^{c}-p_{+}^{c}\right) .
$$

It is instructive to illustrate these equations for a small value of $N$. Choosing $N=10$ and $p_{-}^{c}=p_{+}^{c}=0.159$, the precise $p$-dependence of $P_{+}^{c}(2 \mathrm{~b})$ and of $P^{c}$ (22 $\left.\mathrm{c}\right)$ is depicted in figure 2 . The equality $P_{+}^{c}(p)=1-p_{+}^{c}$ is assumed at the discrete values $p=p_{+}\left(N_{s}\right), N_{s}=0,1, \ldots, N$. For example, as long as $p \leq p_{+}(0)$ holds, $p$ certainty will be smaller than any $p_{+}$bound. As $p$ passes through $p_{+}(0)$, the probability $P_{+}(p)$ jumps down to the value $1-p_{+}^{c}=0.841$. Subsequently $P_{+}^{c}(p)$ rises with $p$ in the range $p_{+}(0)<p<p_{+}(1)$ until, at $p=p_{+}(1)$, the next jump occurs, and so on. The corresponding graph for $P_{-}^{c}(p)$ follows from $P_{+}^{c}(p)$ by reflection 
on the $p=0.5$ axis. The lower, full curve of figure 2 is obtained by combining both according to eqn.(20).

We are interested in the more involved situation where signal and background can no longer be distinguished unambiguously. Instead, a neural network or similar device yields statistical information by tagging signals with efficiency $F_{s}$ and background data with efficiency $F_{b} \quad\left(0 \leq F_{b} \leq 1,0 \leq F_{s} \leq 1\right.$ and, typically, $\left.F_{b} \ll F_{s}\right)$. Applying the network to all $N$ data results in $N^{Y},\left(0 \leq N^{Y} \leq N\right)$ tagged data, composed of $N^{Y}=N_{s}^{Y}+N_{b}^{Y}$, where $N_{s}^{Y}$ are the tagged signals and $N_{b}^{Y}$ are the tagged background data. Of course, the values for $N_{s}^{Y}$ and $N_{b}^{Y}$ are not known. Our task is to determine confidence levels for the signal likelihood $p$ from the sole knowledge of $N^{Y}$. We proceed by writing down the probability density of $N^{Y}$ for given $p$ and, subsequently, generalizing the Clopper-Pearson method.

First, assume fixed $N_{s}$. The probability densities of $N_{s}^{Y}$ and $N_{b}^{Y}$ are binomial and thus the probability density for $N^{Y}$ is given by the convolution

$$
P\left(N^{Y} \mid N_{s}\right)=\sum_{N_{s}^{Y}+N_{b}^{Y}=N^{Y}} b\left(N_{s}^{Y} \mid N_{s}, F_{s}\right) b\left(N_{b}^{Y} \mid N_{b}, F_{b}\right), N_{b}=N-N_{s} .
$$

Summing over $N_{s}$ removes the constraint and the $N^{Y}$-probability density, with $N, p$ fixed, is

$$
P\left(N^{Y} \mid N, p\right)=\sum_{N_{s}=0}^{N} b\left(N_{s} \mid N, p\right) P\left(N^{Y} \mid N_{s}\right) .
$$

For given $p_{-}^{c}, p_{+}^{c}$ and $N^{Y}$, we define confidence limits $p_{-}$and $p_{+}$in analogy with equation (2)

$$
p_{-}^{c}=\sum_{k=N^{Y}}^{N} P\left(k \mid N, p_{-}\right) \quad \text { and } \quad p_{+}^{c}=\sum_{k=0}^{N^{Y}} P\left(k \mid N, p_{+}\right) .
$$

Their meaning is as already outlined by equations (2a-2 2 c). Choosing $F_{s}=0.9, F_{b}=0.2$ and the other parameters as before, figure 3 illustrates these equations for the new situation. The interpretation is as for figure 2 with two remarkable exceptions:

(i) It may happen that eqn.(5) has no solutions $p_{-}\left(N^{Y}\right)$ for certain $N^{Y}=N, N-1, \ldots$ or no solutions $p_{+}\left(N^{Y}\right)$ for certain $N^{Y}=0,1, \ldots$. The reason is that, due to the NN, the result is sufficiently unlikely for all $p$. One may then either decrease $p_{-}^{c}$ or $p_{+}^{c}$ or 
discard the entire analysis. The parameter values of figure 3 are chosen such that there is no solution $p_{+}(0)$. Consequently, there is no longer a range of small $p$-values with $P_{+}^{c}(p)=1$. Such exotic NN output (here $N^{Y}=0$ ) is by definition rare.

(ii) For $F_{s}+F_{b} \neq 1$, the function $P_{-}^{c}(p)$ is no longer a reflection of $P_{+}^{c}(p)$. This is shown in figure 3 , where $P^{c}(p)$ turns out to be no longer symmetric. In fact, on the r.h.s of figure 3 we observe the same feature as in figure 2: The upper and lower curves agree due to $P_{-}(p)=1$ in this range.

In summary, the bounds $\left[p_{-}, p_{+}\right]$obtained with $p_{-}^{c}=p_{+}^{c}=0.159$ guarantee the standard one error bar confidence probability of $68.2 \%$ for every single $p$-value and for almost all $p$ the actual confidence will be better. However, the one-sided bounds cannot be improved without violating the requested confidence probability for some $p$-values. In the same way, bounds calculated with $p_{-}^{c}=p_{+}^{c}=0.023$ ensure the standard two error bar confidence level of $95.4 \%$ or better, and so on. It should be noted that, for $p \neq 0$ and $p \neq 1$, the deviations from the requested confidence probabilities tend to decrease in the limit of large statistics.

\section{Large Data Sets with Few Signals}

We now assume the values of figure 1 to demonstrate the approach in a limit which is of particular interest for experimental high energy physics applications. With $N=26 k$ and $N_{s}=130$ one gets the Clopper-Pearson confidence limits

$$
\begin{aligned}
& 0.00456 \leq p \leq 0.00547 \text { for } p_{-}^{c}=p_{+}^{c}=0.159, \\
& 0.00416 \leq p \leq 0.00595 \text { for } p_{-}^{c}=p_{+}^{c}=0.023 .
\end{aligned}
$$

Next, we assume that the only information about the signals is provided by some NN output, where we use two sets of efficiencies, inspired by [6].

First, we consider $F_{s}=0.5$ and $F_{b}=0.005$. Figure 4 depicts the tag probability density $P\left(N^{Y} \mid N_{s}\right)$, see (3), for three different values of $N_{s}: 0,130$ and 260. There is almost no overlap 
and, consequently, we expect that clear identification of a positive signal can be achieved. For $N_{s}=130$ the central $N^{Y}$ values are located around $N_{s} F_{s}+N_{b} F_{b}=130 F_{s}+(26000-130) F_{b}=$ 194.35. Using $N^{Y}=194$, iteration of equation (5) yields the confidence limits

$$
\begin{aligned}
& 0.00389 \leq p \leq 0.00613 \text { for } p_{-}^{c}=p_{+}^{c}=0.159 \\
& 0.00289 \leq p \leq 0.00729 \text { for } p_{-}^{c}=p_{+}^{c}=0.023 .
\end{aligned}
$$

The computational demand for these results was less than two hours of CPU time on a DEC 3000 Alpha 600 workstation, where it is important to store frequently used coefficients in RAM.

Let us reduce the signal efficiency to $F_{s}=0.1$ and keep the background (in)efficiency unchanged. Figure 5 depicts the new tag probability densities. We find considerable overlap and expect that $p=0$ can no longer be excluded. The central $N^{Y}$ values are now located around 142.35. Using $N^{Y}=142$, iteration of equation (5) gives

$$
\begin{aligned}
& 0.00005 \leq p \leq 0.010 \text { for } p_{-}^{c}=p_{+}^{c}=0.159, \\
& 0.00000 \leq p \leq 0.0153 \text { for } p_{-}^{c}=p_{+}^{c}=0.023 .
\end{aligned}
$$

The latter case should be supplemented by the explicit probability for $p=0$, estimated in the next section.

\section{Signal Probability Distributions}

Equation (5), or of course (2) when applicable, can be used to sandwich the a-posteriori signal probability distribution $F(p)=F\left(p \mid N, N^{Y}\right)$ between lower and upper bounds. Namely, it is easy to see that

$$
F_{1}(p)=1-p_{+}^{c}(p)=\sum_{k=N^{Y}+1}^{N} P(k \mid N, p) \leq F(p) \leq F_{2}(p)=p_{-}^{c}(p)=\sum_{k=N^{Y}}^{N} P(k \mid N, p) .
$$


For their numerical evaluation the sums should be re-written as $F_{2}=1-\sum_{k=0}^{N^{Y}-1} P(k \mid N, p)$ and $F_{1}=F_{2}-P\left(N^{Y} \mid N, p\right)$. Figure 6 depicts these functions for the previously discussed examples $N^{Y}=142$ and 194. Upper and lower bounds are seen close together, such that $F(p)=\left(F_{1}(p)+F_{2}(p)\right) / 2$ would be a reasonable working approximation. The corresponding probability densities are the derivatives with respect to $p$. Their numerical calculation is straightforward when analytical expressions for the derivatives of the binomial coefficients in equation (ब) are used. Figure 7 exhibits the results, $P_{1}\left(p \mid N, N^{Y}\right)$ and $P_{2}\left(p \mid N, N^{Y}\right)$. At $p=0$ the probability densities have $\delta$-function contributions

$$
P_{i}(p)=F_{i}(0) \delta(p)+\ldots,(i=1,2) \text { with } F_{1}(0)=0.136 \text { and } F_{2}(0)=0.156
$$

In addition, or alternatively to the outlined approach, one may be interested to find for $N_{s}=0,1, \ldots, N$ the probabilities that there are $N_{s}$ signals in the data. That could be done using the probability densities $P_{i}\left(p \mid N, N^{Y}\right),(i=1,2)$, but a calculation starting off from $P\left(N^{Y} \mid N_{s}\right)$, equation (3), is far more direct. In particular, it may sometimes be of advantage that $N_{s}$, in contrast to $p$, is a discrete variable. Let us now denote the probability distribution for signals by $F\left(N_{s}\right)=F\left(N_{s} \mid N^{Y}\right)$. Lower and upper bounds are

$$
F_{1}\left(N_{s}\right)=\sum_{k=N^{Y}+1}^{N} P\left(k \mid N_{s}\right) \leq F\left(N_{s}\right) \leq F_{2}\left(N_{s}\right)=\sum_{k=N^{Y}}^{N} P\left(k \mid N_{s}\right) .
$$

Despite of using the same symbols $F, F_{1}$ and $F_{2}$, the functions in equation (6) and (7) are, of course, different. By definition, $F_{2}\left(N_{s}\right)$ is the likelihood that $N_{s}$ signals could have produced the observed $N^{Y}$ or a greater one. Therefore, the likelihood that either of $k=0,1, \ldots, N_{s}$ is correct is less or equal the value $F_{2}\left(N_{s}\right)$, i.e. $F_{2}\left(N_{s}\right)$ is an upper bound of the a-posteriori distribution function $F\left(N_{s}\right)$. Similarly, $1-F_{1}\left(N_{s}\right)$ is an upper bound on the likelihood that either of $k=N_{s}, N_{s}+1, \ldots, N$ is correct. Consequently, $F_{1}\left(N_{s}\right)$ is a lower bound of $F\left(N_{s}\right)$. Figure 8 depicts theses bounds for our standard examples $N^{Y}=142$ and 194 . The similarity with figure 6 is no coincidence, as $p$ determines $N_{s}$ up to fluctuations of order $1 / \sqrt{N}$. Probability densities $P_{i}\left(N_{s} \mid N^{Y}\right)$ are defined by

$$
F_{i}\left(N_{s} \mid N^{Y}\right)=\sum_{k=0}^{N_{s}} P_{i}\left(k \mid N^{Y}\right), \quad(i=1,2)
$$


Defining $F_{i}(-1)=0$, they follow recursively

$$
P_{i}\left(N_{s} \mid N^{Y}\right)=F_{i}\left(N_{s}\right)-F_{i}\left(N_{s}-1\right),\left(N_{s}=0,1, \ldots, N\right) .
$$

Figure 9 exhibits the results. Once the probability densities $P_{i}\left(N_{s} \mid N^{Y}\right), \sum_{N_{s}=0}^{N} P_{i}\left(N_{s} \mid N^{Y}\right)=$ 1 are known, confidence limits can also be calculated from the subsequent generalization of the Clopper-Pearson (2) approach:

$$
p_{-}^{c}=\sum_{N_{s}=0}^{N} P_{2}\left(N_{s} \mid N^{Y}\right) \sum_{k=N_{s}}^{N} b\left(k \mid N, p_{-}\right), \quad p_{+}^{c}=\sum_{N_{s}=0}^{N} P_{1}\left(N_{s} \mid N^{Y}\right) \sum_{k=0}^{N_{s}} b\left(k \mid N, p_{+}\right) .
$$

These equations involve nothing, but weighting the binomial Clopper-Pearson sums with the appropriate probabilities $P\left(N_{s} \mid N^{Y}\right)$. They re-produce the bounds (5) identically, as was numerically checked for our examples of section 3 .

\section{Bayesian Approach}

Our construction invokes the a-priori known fact that the number of signals is in the range $0 \leq N_{s} \leq N$. It is popular (for reviews see [10, 11]), and sometimes quite successful, to make additional assumptions in form of a-priori likelihoods. This can be motivated by a look at figures 2 and 3. For almost all $p$ the confidence is better than the desired $68.2 \%$. If an $a$ priori likelihood is known, the Bayesian approach yields a confidence of precisely $68.2 \%$. The debate is about using a-priori likelihoods in situations where they are not known. Reasonable guesses can apparently be made in many situations. However, false results are obtained when such a guess is in contradiction with the data, which may not always be trivial to uncover. An example is given here.

In our situation, one would be tempted to impose an a-priori likelihood on either $p$ or $N_{s}$. For instance, invoking the maximum-entropy principle [12 leads to constant a-priori probability densities

$$
\rho^{0}(p)=1 \text { or } P^{0}\left(N_{s}\right)=\frac{1}{N+1} \text { for } 0 \leq N_{s} \leq N
$$


For simplicity we focus on the latter case. (Using the result from 13 it would also be straightforward to work out the other one.) As before, $N^{Y}$ is determined by measurements and NN analysis. Under the assumption (10) for $P^{0}\left(N_{s}\right)$, the Bayesian theorem implies the a-posteriori probability

$$
P\left(N_{s} \mid N^{Y}\right)=\text { const. } P\left(N^{Y} \mid N_{s}\right)
$$

with $P\left(N^{Y} \mid N_{s}\right)$ given by equation (3) and the constant follows from the normalization $\sum_{N_{s}=0}^{N} P\left(N_{s} \mid N^{Y}\right)=1$. In case of $N^{Y}=194$ the result agrees very well with that depicted in figure 9. However, this is not true for $N^{Y}=142$, see figure 10, where the probability density of figure 9 is compared with the Bayesian result.

Whereas for strong signal identification the difference with our approach is practically negligible, it is significant for weak (or no) signal identification. The Bayesian probability for $p=0$ is only 0.00215 , implying that the Bayesian distribution function violates the rigorous $F_{1}\left(N_{s}\right)$ bound. The reason is obvious: It is not clear what a-priori probability one should assign to the situation that there is no effect at all. That is why $N_{s}=0$ does not compete on the same level as the numbers $N_{s} \geq 1$. For one of the the situations, we have in mind, no effect at all would mean that there is no top quark. One could assign a finite a-priori probability to this possibility, but whether this is $10 \%, 50 \%$ or $90 \%$ would be highly arbitrary. Actually it does not even work: As the top quark has already be found [7], one may argue in favor of the given a-priori likelihood with the argument that the $N_{s}=0$ is certainly very small. However, this leads to an overestimation of large signal probabilities, as the a-posteriori $N_{s}=0$ likelihood becomes incorrectly re-distributed.

\section{Conclusions}

We have calculated confidence limits of an unknown signal likelihood for the situation where few signals occur in a large number of events. The only input used were neural network efficiencies for tagging signal and background events as well as the number of data the 
network selects. The extension of our approach from the binomial to the multinomial case, i.e. to more than two different types of data (signal and background) is certainly possible.

In typical applications the efficiencies $F_{s}$ and $F_{b}$ may not be known exactly either. Instead, a number of training sets $(j=1, \ldots, J)$ may exist, each giving somewhat different efficiencies

$F_{s}^{j}, F_{b}^{j}$. We think that in this situation a bootstrap type of approach [8] can be applied and that the probability density (8) provides a suitable starting point. We can linearly combine different probability densities to an ultimate one

$$
P_{i}\left(N_{s}\right)=J^{-1} \sum_{j=1}^{J} P_{i}^{j}\left(N_{s} \mid N_{j}^{Y}\right),(i=1,2),
$$

and proceed with $P_{i}\left(N_{s}\right)$ as discussed in section 4 .

Finally, to involve conjectured a-priori likelihoods may in many situations be unavoidable and, actually, be quite successful. In our case: When a clear, positive signal identification is possible, we find practically no difference between a Bayesian maximum entropy and our approach. However, our example of weak signal identification shows that a-priori likelihoods are better avoided when a rigorous alternative exists.

\section{References}

[1] C.J. Clopper and E.S. Pearson, Biometrika 26 (1934) 404; S. Brandt, Statistical and Computational Methods in Data Analysis (North-Holland, 1983).

[2] L. Lönnblad, C. Peterson and T. Rögnvaldsson, Comp. Phys. Commun. 81 (1994) 187; Nucl. Phys. B 349 (1991) 675.

[3] K.H. Becks, F. Block, J. Dress, P. Langefeld and F. Seidel, Nucl. Instrum. Methods A $329(1993) 501$.

[4] G. Stimpf-Abele and P. Yepes, Comp. Phys. Commun. 78 (1993) 1.

[5] M.D. Richard and R.P. Lippmann, Neural Comput. 3 (1991) 461. 
[6] B.A. Berg, Comp. Phys. Commun. 98 (1996) 35.

[7] CDF Collaboration, F. Abe et al., Phys. Rev. Lett. 74 (1995) 2626; D0 Collaboration, S. Abachi et al., Phys. Rev. Lett. 74 (1995) 2632.

[8] B. Efron, J. Am. Stat. Assoc. 82 (1987) 171.

[9] W.H. Press, B.P. Flannery, S.A. Teukolsky and W.T. Vetterling, Numerical Recipes (Cambridge University Press, 1988).

[10] T.J. Loredo, From Laplace to Supernova SN1987A: Bayesian Inference in Astrophysics, in: Maximum Entropy and Bayesian Methods, P.F. Fougère, ed. (Kluwer Academic Publishers, Dortrecht, 1990) pp. 81-142.

[11] A. Gelman, J. B. Carlin, H.S. Stern and D.B. Rubin, Bayesian Data Analysis (Chapman \& Hall, 1995).

[12] E.T. Jaynes, Proc. IEEE 70 (1982) 939.

[13] Ref. 11, p.29. 


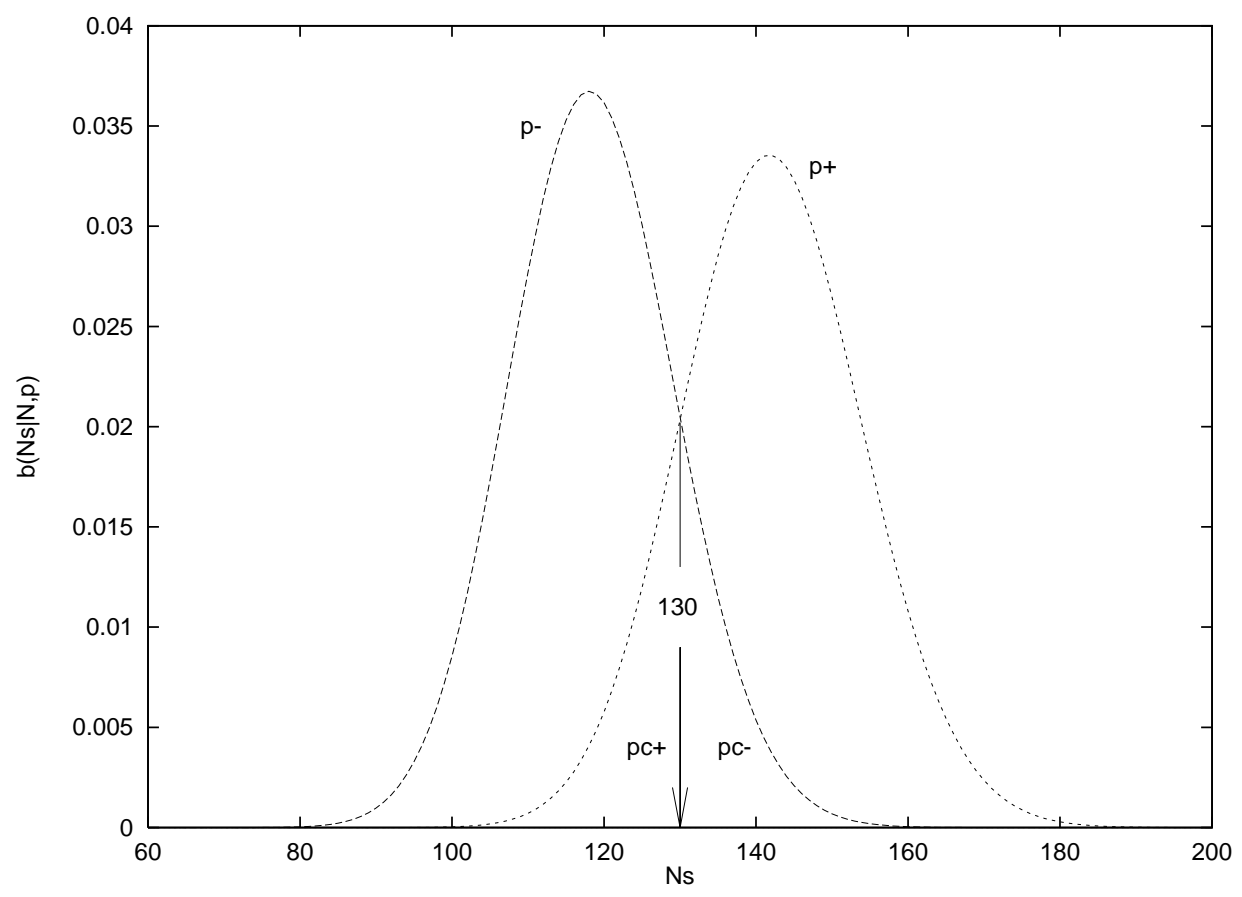

Figure 1: Binomial probability densities corresponding to solving equations (2) for $p_{+}$and $p_{-}$with $p_{-}^{c}=p_{+}^{c}=0.159, N=26 k$ and $N_{s}=130$.

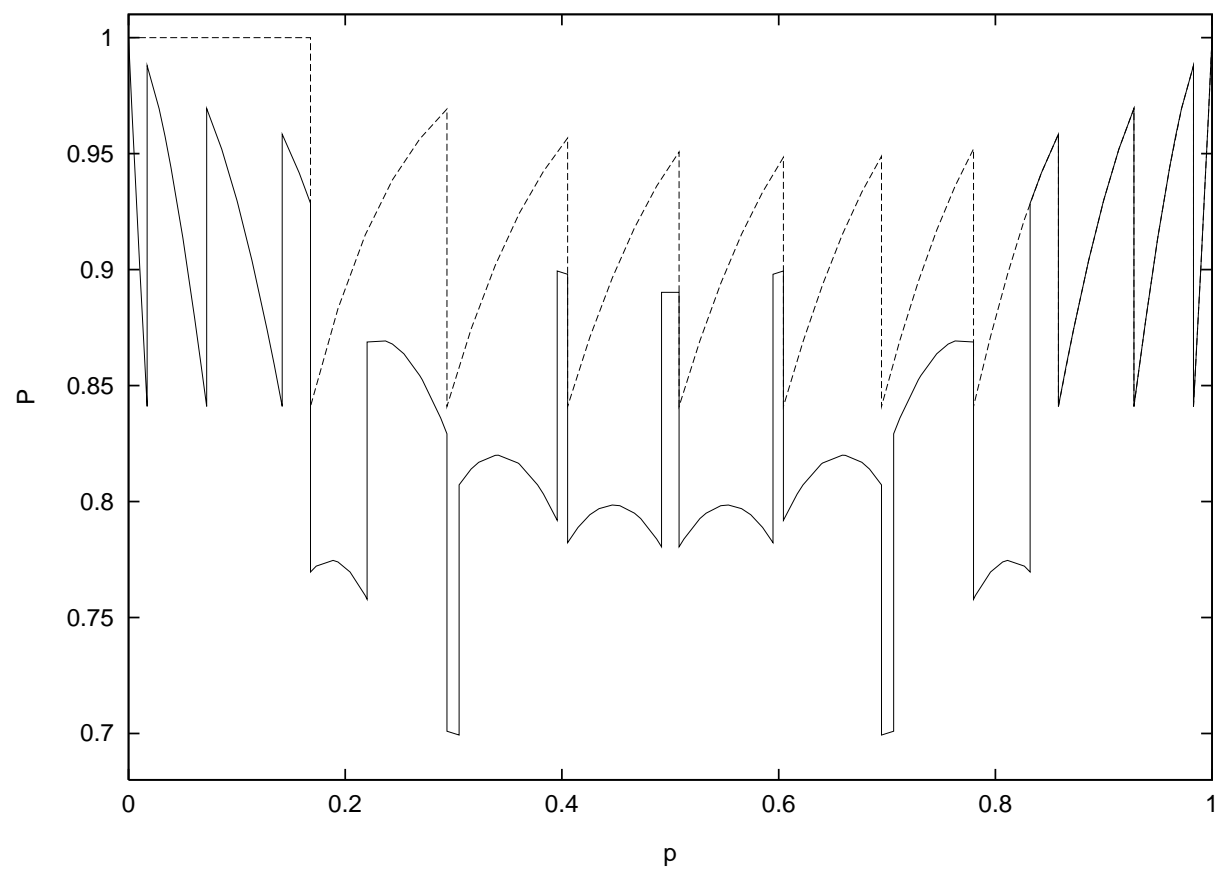

Figure 2: Confidence likelihoods for the Clopper-Pearson bounds (2). The parameters $N=$ 10 and $p_{-}^{c}=p_{+}^{c}=0.159$ are used. Upper, broken line: Confidence likelihood $P=P_{+}^{c}(p)$ (22 $\mathrm{b}$ ) versus the true signal probability $p$. Lower, full line: Confidence likelihood $P=P^{c}(p)$ (2) versus $p$. 


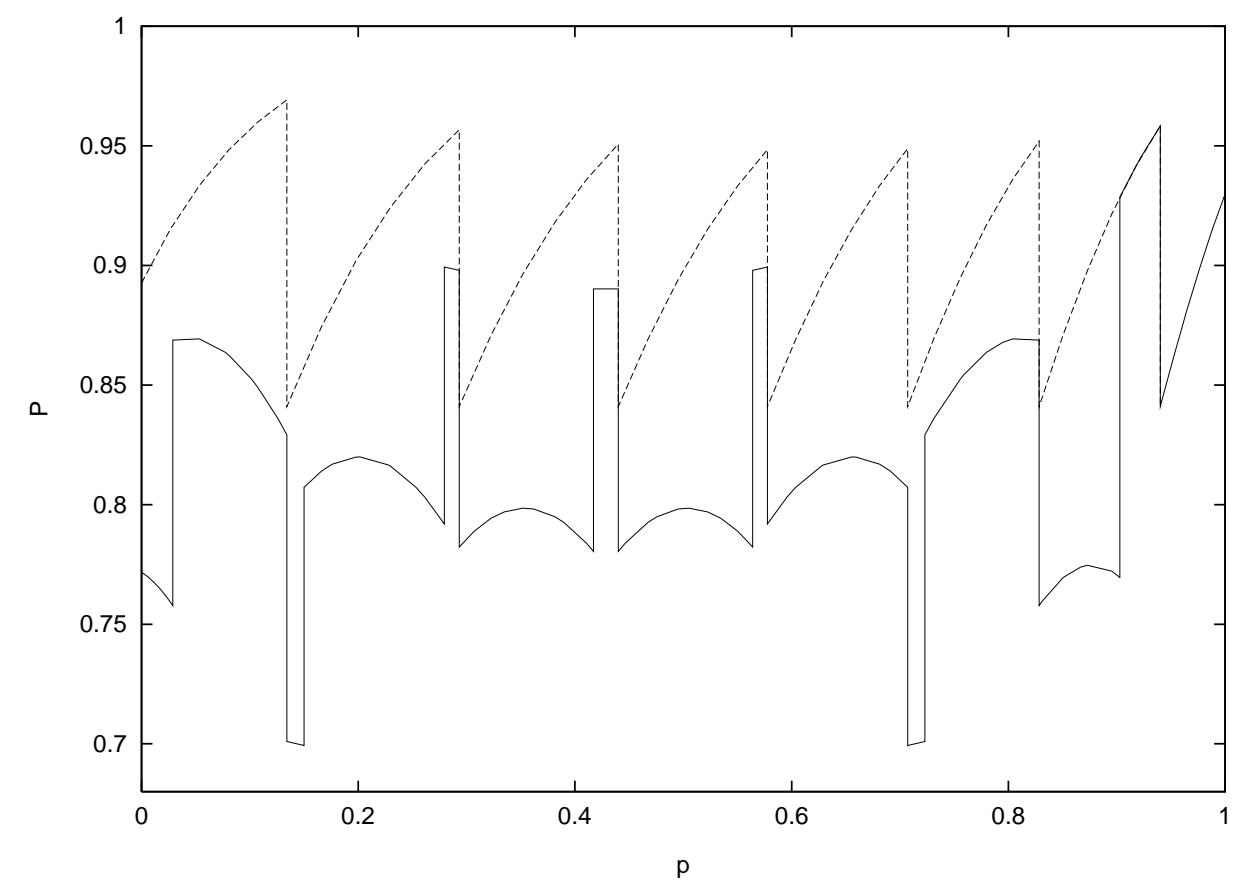

Figure 3: Confidence likelihoods for the generalized Clopper-Pearson bounds (5). The parameters $F_{s}=0.9, F_{b}=0.2$ and those of figure 2 are used. Upper, broken line: Confidence likelihood $P=P_{+}^{c}(p)(2 \mathrm{~b})$ versus the true signal probability $p$. Lower, full line: Confidence likelihood $P=P^{c}(p)($ (5) $)$ versus $p$.

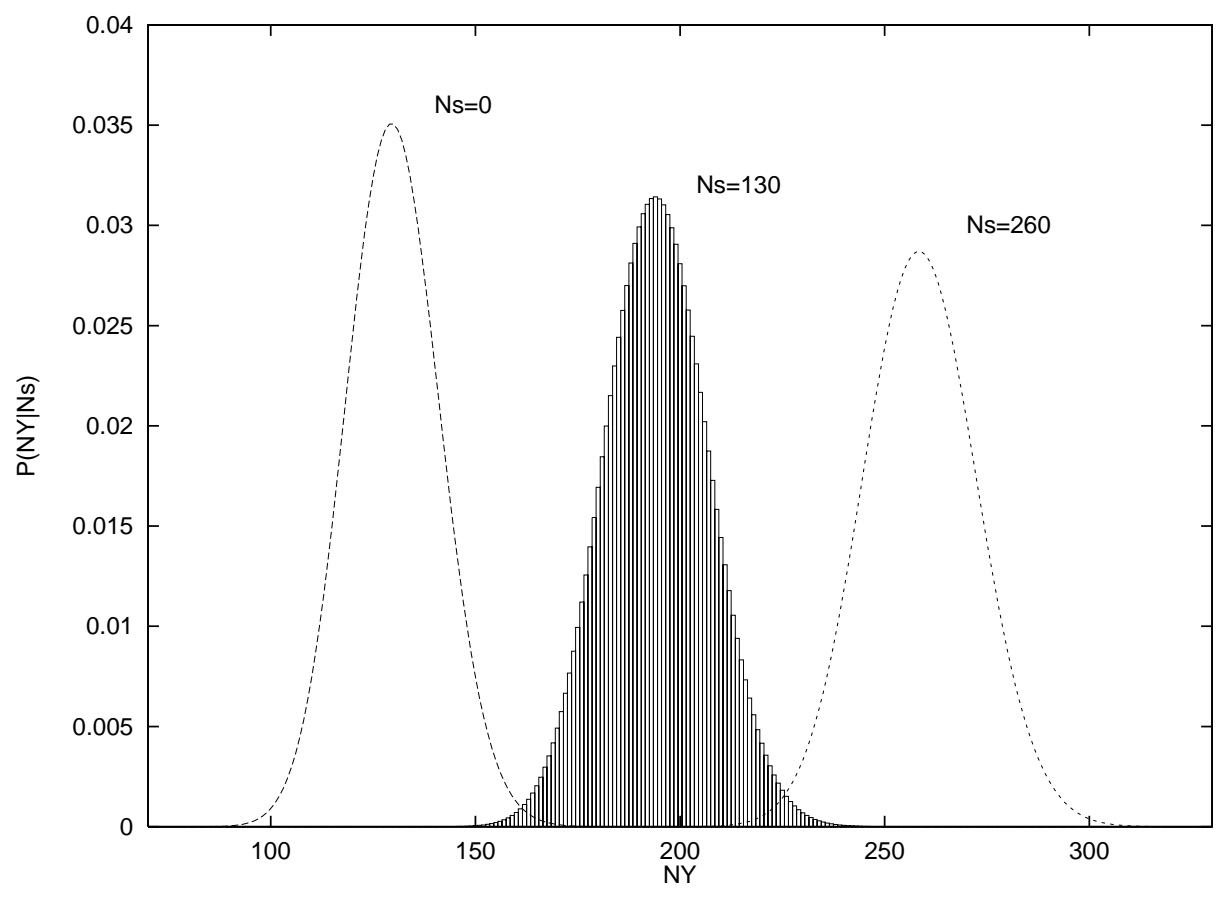

Figure 4: Probability densities to get $N^{Y}$ data from the NN employing the efficiencies $F_{b}=0.005, F_{s}=0.5$ and assuming $N_{s}$ signals in the original $26 k$ data set. 


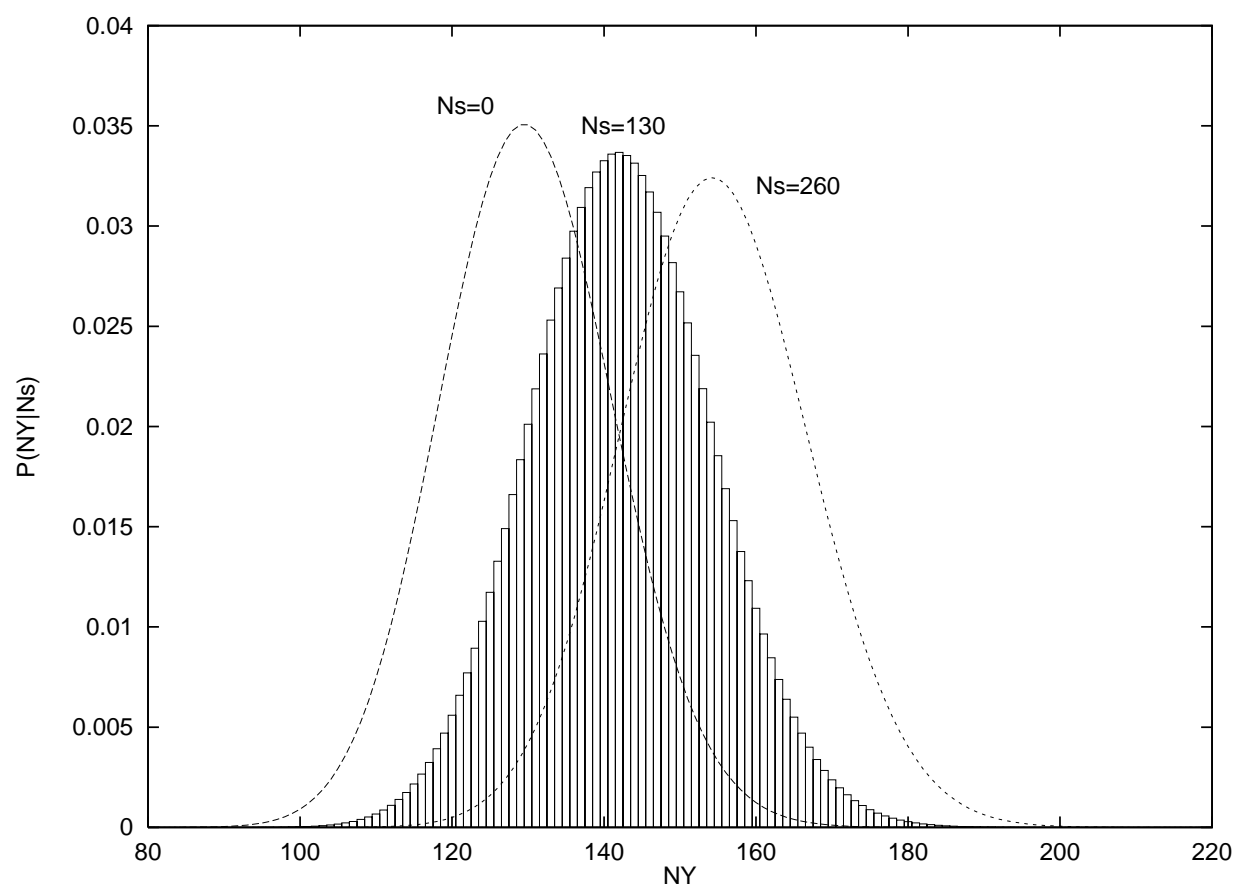

Figure 5: As figure 4, but with signal network efficiency $F_{s}=0.1$.

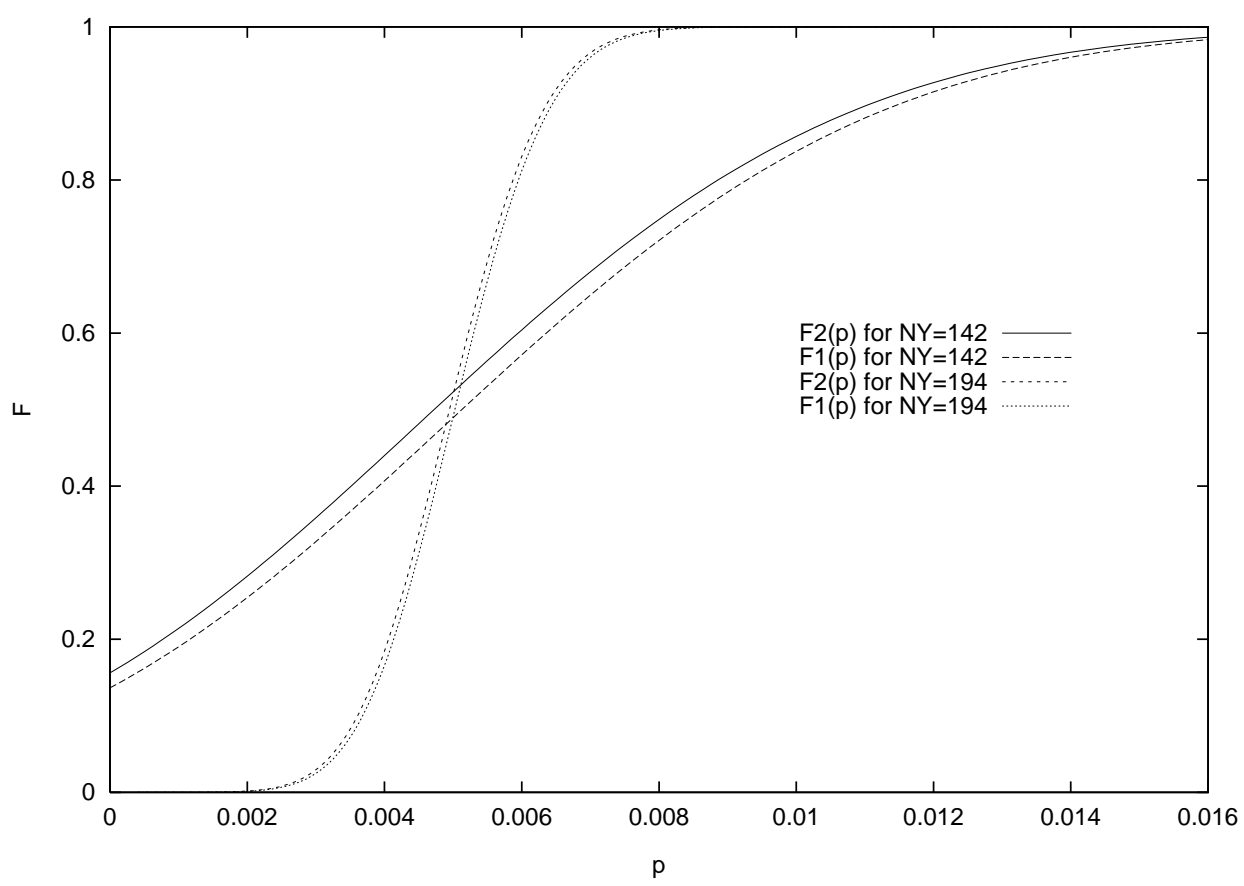

Figure 6: A-posteriori signal probability distributions (upper and lower bounds). 


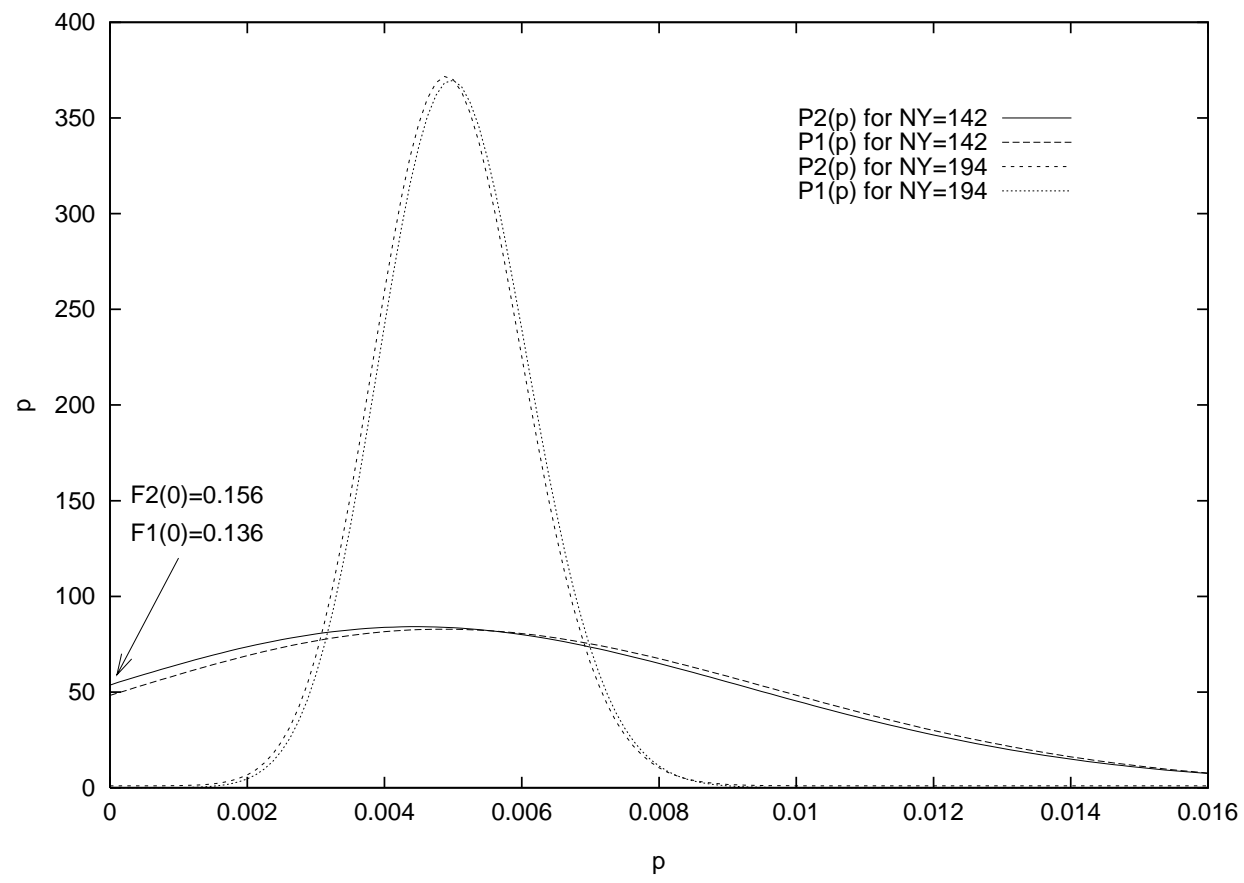

Figure 7: A-posteriori signal probability densities (corresponding to upper and lower bounds of the distribution functions in figure 6).

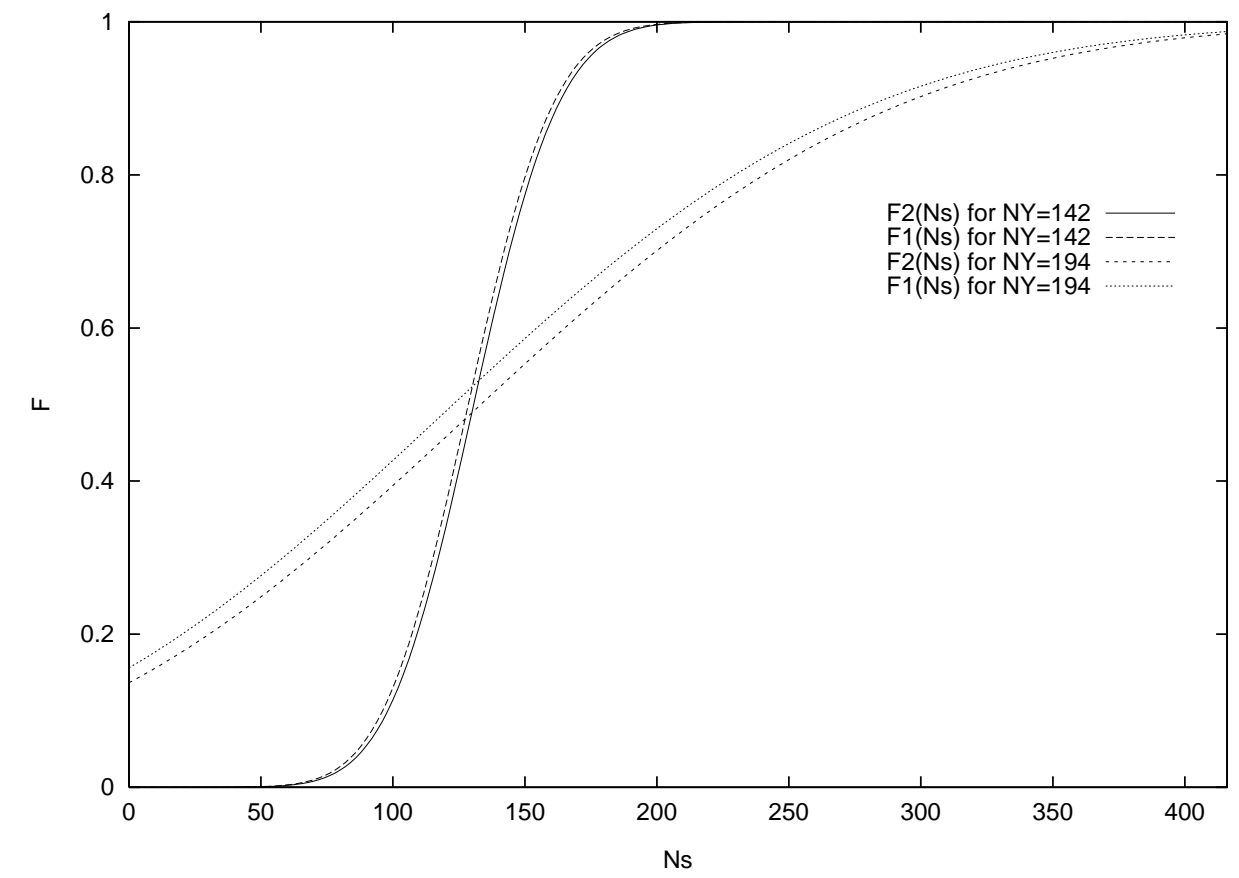

Figure 8: A-posteriori distributions for the number of signals (upper and lower bounds). 


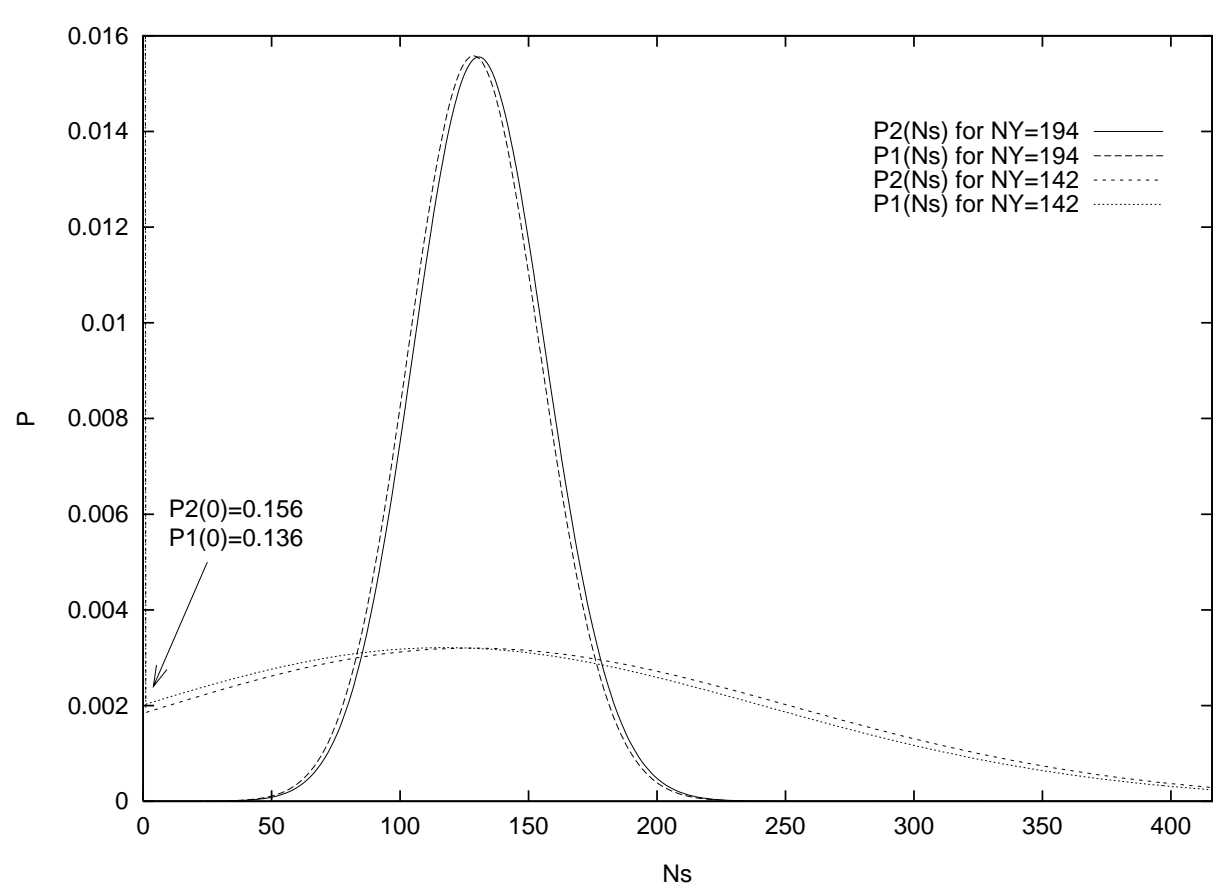

Figure 9: A-posteriori probability densities for the number of signals (corresponding to upper and lower bounds of the distribution functions in figure 8).

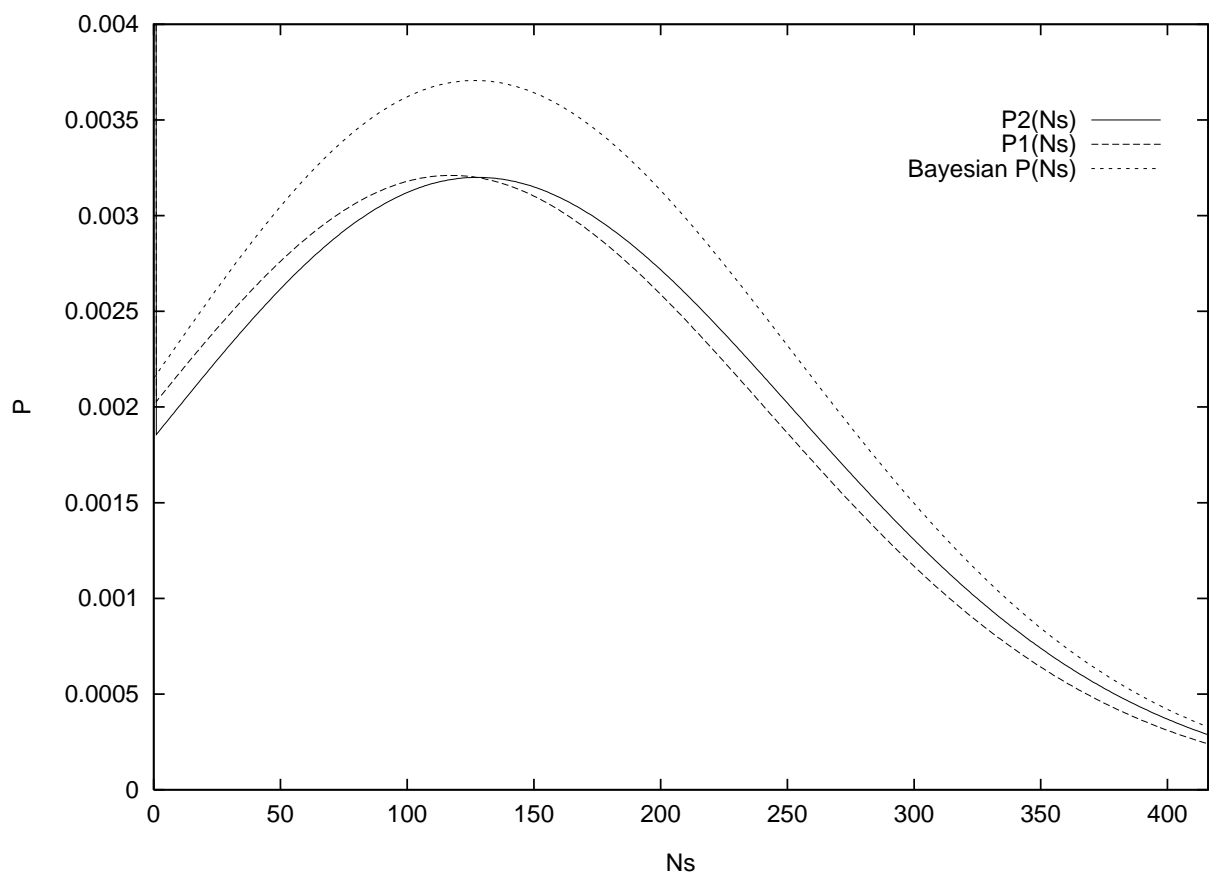

Figure 10: $N^{Y}=142$ : Comparison of the Bayesian (maximum entropy) a-posteriori probability density $P\left(N_{s}\right)$ with $P_{1}\left(N_{s}\right)$ and $P_{2}\left(N_{s}\right)$ of figure 9 . 


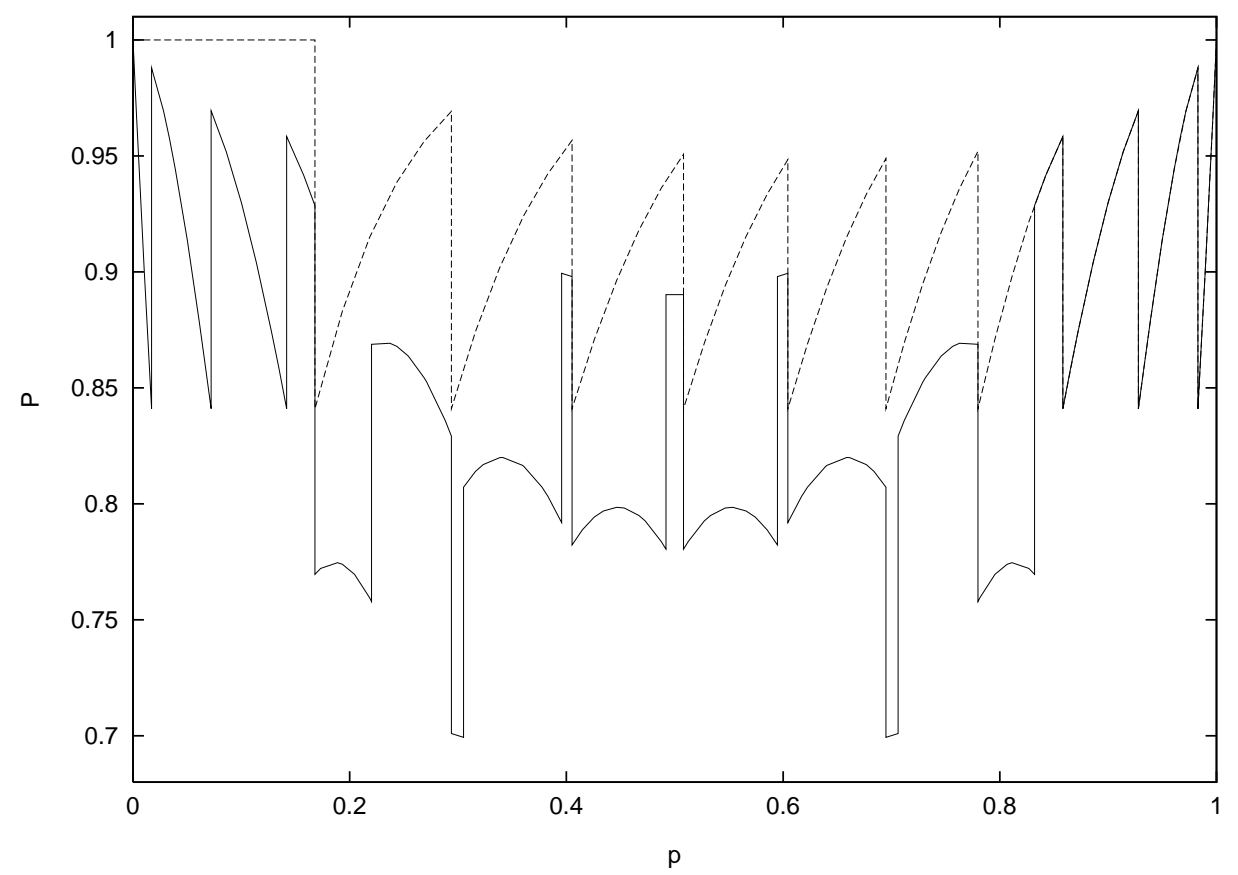




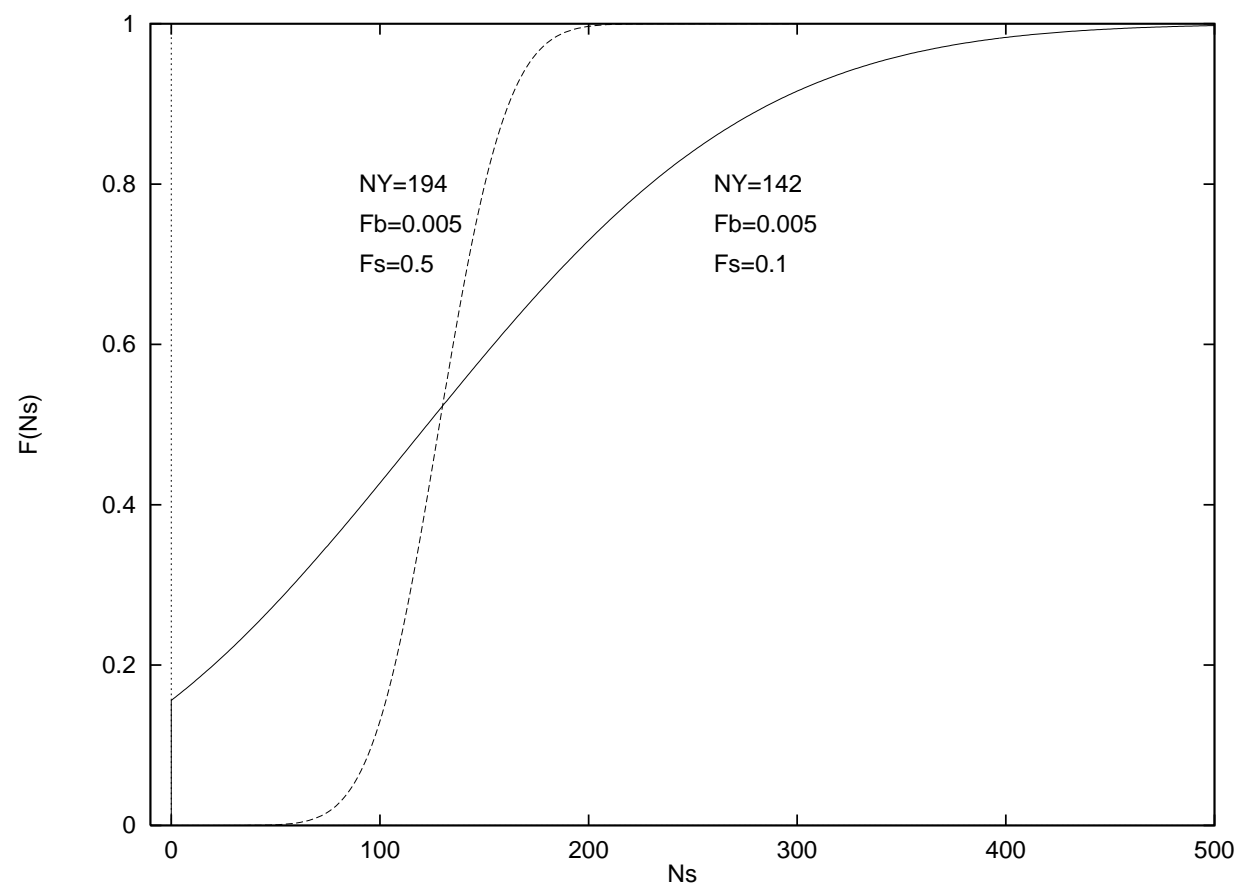




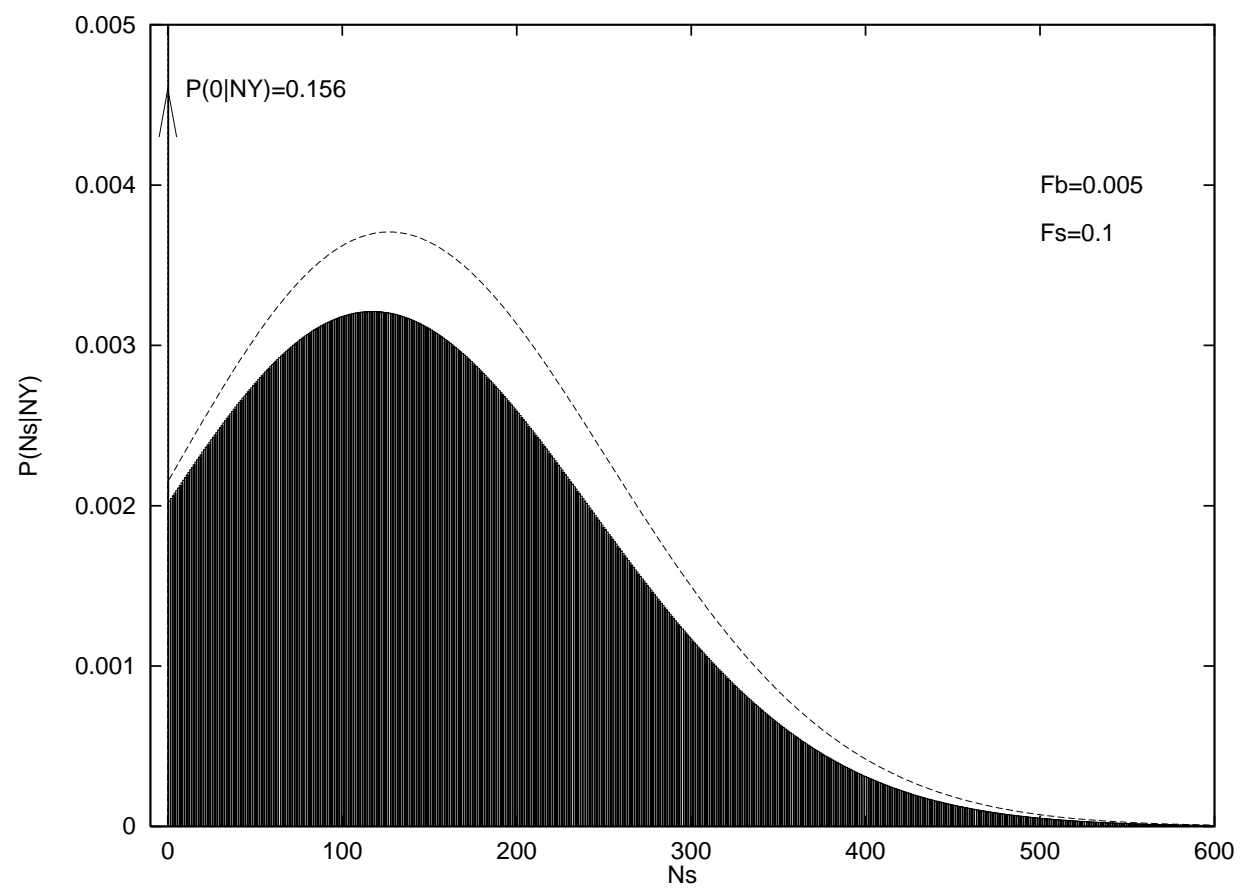




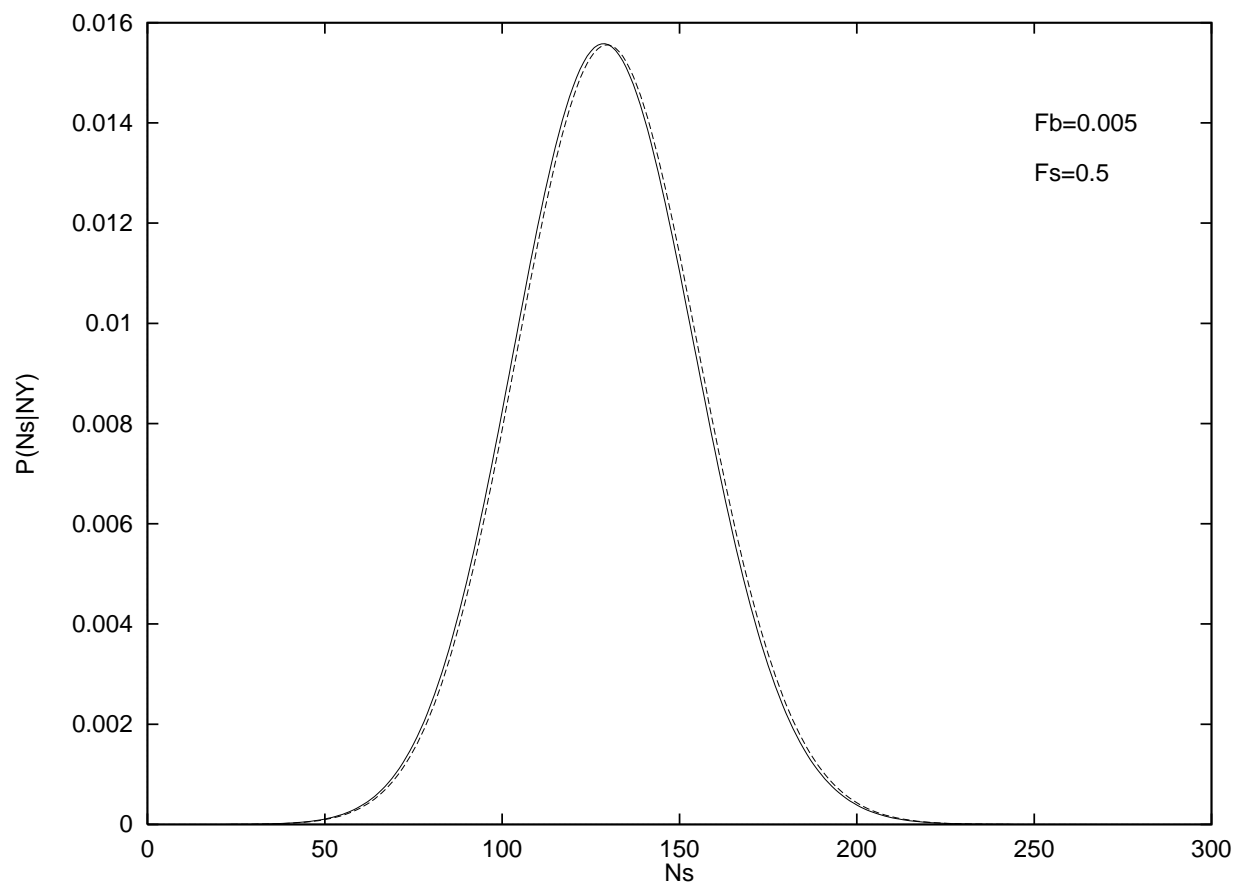

